\title{
Analyticity Constraints on Unequal-Mass Regge Formulas*
}

\author{
Marvin L. Goldberger and C. Edward Jones \\ Palmer Physical Laboratory, Princeton University, Princeton, New Jersey
}

(Received 27 May 1966)

\begin{abstract}
A Regge-pole formula is derived for the elastic scattering of two unequal-mass particles that combines desirable $l$-plane analytic properties (i.e., a simple pole at $l=\alpha$ in the right-half $l$ plane) and Mandelstam analyticity. It is verified that such a formula possesses the standard asymptotic Regge behavior $u^{\alpha(s)}$ even in regions where the cosine of the scattering angle of the relevant crossed reaction may be bounded. The simultaneous requirements of $l$-plane and Mandelstam analyticity enforce important constraints, and the consistency of these constraints is studied. These considerations lead to the appearance of a "background" term proportional asymptotically to $u^{\alpha(0)-1}$ which has no analog in the equal-mass problem. We also conclude that a necessary condition for consistency is $\alpha(\infty)<0$.
\end{abstract}

\section{INTRODUCTION}

$T$ HE description of very high energy scattering processes in terms of Regge poles has been widely discussed. In practice one is, of course, concerned with experiments carried out at energies which are probably too low for the simplest asymptotic forms to be accurate. It is consequently of importance to use representations in which the proper analyticity, threshold behavior, contributions of Regge poles in all three channels, and background (i.e., non-Regge) terms are taken into account as well as possible. Several efforts in this direction have been made for equal-mass scattering processes. ${ }^{1}$ The present paper is devoted in part to a reexamination of such representations with particular attention to analyticity and consistency. Our principal concern is, however, to discuss the surprisingly nontrivial extension of these ideas to processes involving unequal masses (e.g., $\pi-N$ scattering). The directly related question of asymptotic behavior in situations where the cosine of the scattering angle in the crossed reaction is not large is explored in detail.

Stated briefly, we have found the following results: (1) The requirements of analyticity implied by the Mandelstam representation impose important constraints on a representation of scattering amplitudes in terms of Regge poles and "background terms." These constraints imply the necessary condition that $\alpha(\infty)<0$ and they require, in the unequal-mass problem, a "background" term proportional to $u^{\alpha(0)-1}$. (2) The characteristic power-law behavior of amplitudes (e.g., $A \sim u^{\alpha(s)}$, with $s$ and $u$ the usual variables) persists even in those unequal-mass situations where a too naive application of Regge analysis does not allow a prediction.

In Sec. II we discuss the equal-mass problem to determine a Regge-like representation consistent with analyticity, one whose background term is of known size. The corresponding and rather more complicated discussion for the unequal-mass case is given in Sec. III. The question of asymptotic behavior is treated in Sec.

\footnotetext{
* Work supported by the U. S. Air Force Office of Research, Ai Research and Development Command under Contract No. AF49(638)-1545.

${ }^{1}$ G. F. Chew and C. E. Jones, Phys. Rev. 135, B208 (1964).
}

IV and some general considerations, speculations, and suggestions are given in Sec. V.

\section{EQUAL-MASS PROBLEM}

We consider the scattering of two spinless particles of equal mass. We confine our attention to that portion of the amplitude coming from the $s-t$ double spectral function:

$$
A(s, t)=\frac{1}{\pi^{2}} \iint \frac{d s^{\prime}}{s^{\prime}-s} \frac{d t^{\prime}}{t^{\prime}-t} \rho\left(s^{\prime}, t^{\prime}\right),
$$

where $s$ is the square of the total energy and $t$ is the negative squared momentum transfer. In the special case of potential scattering, Eq. (2.1) gives the complete scattering amplitude, whereas in the relativistic case the contribution from the other two spectral regions is not included. We treat each spectral function separately so we can discuss both cases at once.

In addition to the requirement of Mandelstam analyticity expressed in Eq. (2.1), we shall also assume that the particle-wave amplitude $a(s, l)$ is meromorphic in the angular-momentum plane in a region that includes Rel $>-\frac{1}{2}+\epsilon$, where $0<\epsilon<\frac{1}{2}$. We maintain a discreet silence on the question of moving branch points in the $l$ plane. We make a few comments on the subject in Sec. $\mathrm{V}$. The consequences of requiring both Mandelstam and $l$-plane analyticity will now be explored. Our object is to derive a formula in which the Regge-pole terms have the correct analyticity and where any correction terms are no larger asymptotically than the usual Regge background, that is, bounded by $t^{-\frac{1}{2}+\epsilon}$ for large $t$.

We use the Mandelstam ${ }^{2}$ version of the ReggeSommerfeld-Watson representation:

$$
\begin{aligned}
A(\nu, t)=B(\nu, t)+\sum_{i} \gamma_{i}(\nu)(\nu)^{\alpha_{i}(\nu)} & \\
& \times Q_{-1-\alpha_{i}(\nu)}\left(-1-\frac{t}{2 \nu}\right),
\end{aligned}
$$

where we have introduced for economy of writing the square of the center-of-mass momentum $\nu=\frac{1}{4} s-m^{2}$. In

${ }^{2}$ S. Mandelstam, Ann. Phys. (N. Y.) 19, 254 (1962). 
potential theory, $\nu=2 m E$, where $E$ is the center-of-mass energy and $m$ is the reduced mass. The first term, $B(\nu, t)$, is the background term, defined more precisely below; $\gamma_{i}(\nu)(\nu)^{\alpha_{i}(\nu)}$ is related to the actual residue $\beta_{i}(\nu)$ of a Regge pole in the complex angular-momentum plane at $l=\alpha_{i}(\nu)$ according to $\gamma_{i}(\nu)(\nu)^{\alpha_{i}(\nu)}=\left(2 \alpha_{i}(\nu)+1\right) \beta_{i}(\nu) /$ $\cos \pi \alpha_{i}(\nu) ; Q_{-1-\alpha(-z)}$ is the Legendre function of the second kind and $z=1+t / 2 \nu$ is the cosine of the scattering angle. The virtue of the representation (2.2) over the more familiar one using $P_{\alpha}(-z)$ is that while $P_{\alpha}$ has the Regge pole at $l=\alpha$, it also possesses a spurious pole at $l=-\alpha-1 ; Q_{-1-\alpha}$ has only the pole at $l=\alpha$. We separate from the amplitude and write explicitly all those Regge poles which appear in the region $\mathrm{Rel}>-\frac{1}{2}$ $+\epsilon$ for any value of the energy above threshold, $\nu>0$. The background term $B(\nu, t)$ is thus defined uniquely to be the analytic function of $\nu$ and $t$ which is the difference between $A(\nu, t)$ and these selected poles. The function $B(\nu, t)$ is equivalent to a line integral in the $l$ plane which, at a given value of $\nu$, runs from $-\frac{1}{2}+\epsilon-i \infty$ to $-\frac{1}{2}+\epsilon$ $+i \infty$, detouring to the left of any pole which for some $\nu$ above threshold reaches the region $\mathrm{Rel}>-\frac{1}{2}+\epsilon$. We may therefore assert that $B(\nu, t)$ is an analytic function of $\nu$ and $t$ with the property

$$
B(\nu, t)<\text { const } \times t^{-\frac{1}{2}+\epsilon}, \quad(t \rightarrow \infty)
$$

for all positive $\nu$ above threshold. In what follows we shall suppress the summation sign and the index $i$ in Eq. (2.2).

It is well known that neither $B(\nu, t)$ nor the $Q$ functions in Eq. (2.2) have the correct Mandelstam analyticity as expressed by Eq. (2.1). It can be shown explicitly in potential theory that a cancellation takes place between the terms, and that the proper double spectral function is obtained. In what follows we assume that such a cancellation always occurs. We proceed to write a fixed $t<0$ dispersion relation ${ }^{3}$ for $A(\nu, t)$ :

$$
\begin{aligned}
A(\nu, t)= & \frac{1}{\pi} \int_{0}^{\infty} \frac{d \nu^{\prime}}{\nu^{\prime}-\nu} \operatorname{Im} A\left(\nu^{\prime}, t\right) \\
= & \frac{1}{\pi} \int_{0}^{\infty} \frac{d \nu^{\prime}}{\nu^{\prime}-\nu} \operatorname{Im} B\left(\nu^{\prime}, t\right)+\frac{1}{\pi} \int_{0}^{\infty} \frac{d \nu^{\prime}}{\nu^{\prime}-\nu} \\
& \times \operatorname{Im}\left[\gamma\left(\nu^{\prime}\right)\left(\nu^{\prime}\right)^{\alpha\left(\nu^{\prime}\right)} Q_{-1-\alpha\left(\nu^{\prime}\right)}\left(-1-\frac{t}{2 \nu^{\prime}}\right)\right] ;
\end{aligned}
$$

in the following we shall not show the argument of $\alpha$ but if it appears under an integral, it will always be a function of $\nu^{\prime}$. Possible subtractions in Eq. (2.4) are disregarded because they do not affect the subsequent

\footnotetext{
${ }^{3}$ The use of a fixed- $t$ dispersion relation to discuss the Regge asymptotic form for values of $\nu$ other than $\nu>0$ was apparently first described by $\mathbf{H}$. Cheng (unpublished). It is further discussed by Squires in Complex Angular Momentum and Particle Physics (W. A. Benjamin, Inc., New York, 1963), p. 13. Squires does not, however, address the question of full Mandelstam analyticity.
}

discussion. Note that we require the representation (2.2) only in the region for which it was originally defined, namely, $\nu>0, t<0$. We now study the integral over the Regge pole in Eq. (2.4), call it $A_{R}(\nu, t)$. It manifestly has the proper $s$ cut and we wish to examine its $t$ analyticity.

The Regge term in Eq. (2.4) may be written as a contour integral and then may be easily evaluated. It becomes

$$
\begin{array}{r}
A_{R}(\nu, t)=\frac{1}{2 \pi i} \int \frac{d \nu^{\prime}}{C} \gamma\left(\nu^{\prime}\right)\left(\nu^{\prime}\right)^{\alpha} Q_{-1-\alpha}\left(-1-\frac{t}{2 \nu^{\prime}}\right) \\
=\gamma(\nu)(\nu)^{\alpha} Q_{-1-\alpha}\left(-1-\frac{t}{2 \nu}\right) .
\end{array}
$$

The contour $C$ encloses the real $\nu^{\prime}$ axis in a clockwise direction. The equivalence of the contour integral in Eq. (2.5) to the corresponding term in Eq. (2.4) which involves the imaginary part follows from the reality of the integrand just below $\nu=0$. It is assumed here that $\alpha$ and $\gamma$ have only the usual threshold branch point; the function $Q_{-1-\alpha}(-z)$ has branch points at $z= \pm 1$ and $z=\infty$. Since, in fact, the integrand of Eq. (2.5) has no singularities at all below $\nu=0(t<0)$, we can completely unwrap the contour $C$ and thus obtain just a contribution from the pole at $\nu^{\prime}=\nu$.

Although the function $\gamma(\nu)(\nu)^{\alpha} Q_{-1-\alpha}(-1-t / 2 \nu)$ has the correctly located $\nu$ cut for $t<0$, the $\nu$ cut is wrong for $t>0$. Furthermore, the branch cut in $t$ does not start in the right place, that is, at the place required by the Mandelstam analyticity. Since in practice where one is concerned with the amplitude in a crossed channel (with $t>0, \nu<0$ ) it is important to get the analyticity right, we therefore proceed to correct these deficiencies. First, we analytically continue Eq. (2.5) to the region $\nu<0$ and then to $t \gg 0$ above the $t$ cut. We can thus determine the absorptive part $D_{t}(t, \nu)$ in this region to be

$$
\begin{aligned}
D_{t}(t, \nu)=\operatorname{Im} A_{R}(\nu, t)=-\gamma(\nu)(-\nu)^{\alpha} \sin \pi \alpha \\
\quad \times Q_{-1-\alpha}(-1-t / 2 \nu) . \quad \nu<0, \quad t \gg 0 .
\end{aligned}
$$

We see that $D_{t}(\nu, t)$ has a cut from $\nu=-\frac{1}{4} t$ to $\nu=-\infty$, which is at variance with the Mandelstam analyticity, Eq. (2.1). We therefore explicitly remove this unwanted cut and define a corrected $D_{t}$, call it $\widetilde{D}_{t}$ :

$$
\begin{gathered}
\widetilde{D}_{t}=D_{t}-\frac{1}{2} \int_{-\infty}^{-t / 4} \frac{d \nu^{\prime}}{\nu^{\prime}-\nu} \gamma\left(\nu^{\prime}\right)\left(-\nu^{\prime}\right)^{\alpha} \cdot \sin \pi \alpha P_{\alpha}\left(-1-t / 2 \nu^{\prime}\right) \\
-\frac{1}{\pi} \int_{0}^{d /\left(t-t_{0}\right)} \frac{d \nu^{\prime}}{\nu^{\prime}-\nu} \operatorname{Im}\left[-\gamma\left(\nu^{\prime}\right)\left(\nu^{\prime}\right)^{\alpha}\right. \\
\left.\times \sin \pi \alpha Q_{-1-\alpha}\left(+1+t / 2 \nu^{\prime}\right)\right]
\end{gathered}
$$

In writing Eq. (2.7) we have also corrected $D_{t}(t, \nu)$ in order to get the correct boundary of the double spectral function. This is done by means of the second integral in Eq. (2.7) [ $d$ is a constant and $t_{0}$ is the $t$ threshold]. 
Our final form for the Regge-pole term $R(\nu, t)$ is then given by

$$
R(\nu, t)=\frac{1}{\pi} \int_{t_{0}}^{\infty} \frac{d t^{\prime}}{t^{\prime}-t} \widetilde{D}_{t}\left(t^{\prime}, \nu\right),
$$

where the integral in Eq. (2.8) is defined, if necessary, by means of analytic continuation in $\alpha$. The Regge term $R(\nu, t)$ now has the correct Mandelstam analyticity. A formula similar to Eq. (2.8) was first discussed by Chew and Jones. ${ }^{1}$

We come now to the important question of the consistency of the Mandelstam representation and meromorphy in the $l$ plane in the region Rel $>-\frac{1}{2}+\epsilon$. The fixed $t(<0)$ dispersion relation Eq. (2.4) was written on the assumption that there was complete consistency -in particular, it assumes that the background term and the Regge-pole term conspire in such a way as to give the correct Mandelstam $\nu$ cut for any value of $t$, not just the negative $t$ values for which the dispersion relation was originally written. This implies that the "error" ln the pole term is of background magnitude and it is just this consistency which we now check.

To study the consistency question we write the onedimensional dispersion relation Eq. (2.4) as a twodimensional one as follows:

$$
\begin{aligned}
A(\nu, t)= & \frac{1}{\pi^{2}} \iint \frac{d \nu^{\prime}}{\nu^{\prime}-\nu} \frac{d t^{\prime}}{t^{\prime}-t} \\
& \times\left\{\operatorname{Im} B\left(\nu^{\prime}, t^{\prime}\right)\right\}_{t}+\frac{1}{\pi^{2}} \iint \frac{d \nu^{\prime}}{\nu^{\prime}-\nu} \frac{d t^{\prime}}{t^{\prime}-t} \\
& \times\left\{\operatorname{Im}\left[\gamma\left(\nu^{\prime}\right)\left(\nu^{\prime}\right)^{\alpha} Q_{-1-\alpha}\left(-1-\frac{t^{\prime}}{2 \nu^{\prime}}\right)\right]\right\}_{t},
\end{aligned}
$$

where $\{\cdots\}_{t}$ means the imaginary part which is obtained when $t$ is continued from the negative values for which the quantities $\operatorname{Im} B\left(\nu^{\prime}, t\right)$ and $\operatorname{Im}\left[\gamma\left(\nu^{\prime}\right) \nu^{\prime \alpha} Q_{-1-\alpha}\right.$ $\left.\times\left(-1-t / 2 \nu^{\prime}\right)\right]$ were originally defined. The region of integration in Eq. (2.9) is presumed to be just over the proper double spectral function boundary-we have thus assumed that the requisite cancellation does occur. The second term in Eq. (2.9) is just our previously defined $R(\nu, t)$, Eq. (2.8). We now define a correction term $C(\nu, t)$ given by

$$
C(\nu, t)=R(\nu, t)-\gamma(\nu)(\nu)^{\alpha} Q_{-1-\alpha}(-1-t / 2 v) .
$$

Recalling that according to Eq. (2.2), $A(\nu, t)=B(\nu, t)$ $+\gamma(\nu) \nu^{\alpha} Q_{-1-\alpha}(-1-t / 2 \nu)$, we find from Eq. (2.9) that

$C(\nu, t)=B(\nu, t)-\frac{1}{\pi^{2}} \iint \frac{d \nu^{\prime}}{\nu^{\prime}-\nu} \frac{d t^{\prime}}{t^{\prime}-t}\left\{\operatorname{Im} B\left(\nu^{\prime}, t^{\prime}\right)\right\}_{t}$.

Thus if $\nu>0$, the consistency of our assumed Mandelstam and $l$-plane analyticity leads to the statement

$$
C(\nu, t)<\text { const } \times t^{-\frac{1}{2}+\epsilon}, t \rightarrow \infty .
$$

This follows because both terms on the right of Eq. (2.11) are of Regge background size for $\nu>0$ and large $t$.

We may now check the requirement expressed by Eq. (2.12). By utilizing the well-known representation

$$
\begin{aligned}
Q_{\beta}(z)=\frac{1}{2} \int_{-1}^{+1} d z^{\prime} \frac{P_{\beta}\left(z^{\prime}\right)}{z-z^{\prime}} & \\
& +\frac{1}{\pi} \sin \pi \beta \int_{-\infty}^{-1} d z^{\prime} \frac{Q_{\beta}\left(-z^{\prime}\right)}{z^{\prime}-z},
\end{aligned}
$$

we may extract an explicit $Q_{-1-\alpha}(-1-t / 2 v)$ from $R(\nu, t)$ as given by Eq. (2.8) and write an exact expression for $C(\nu, t)$. It is clear, however, that to within terms asymptotically of order $t^{-1}$, the large $t$ behavior of $C(\nu, t)$ is the same as that of $\widehat{D}_{t}(t, \nu)-D_{t}(t, \nu)$. A straightforward estimate of Eq. (2.7) leads to ${ }^{3 \mathrm{a}}$

$$
\widetilde{D}_{t}(t, \nu)-D_{t}(t, \nu) \sim C_{1} t^{\alpha(\infty)}+C_{2} t^{-3 / 2},
$$

where $C_{1}$ and $C_{2}$ are functions of $\nu(\nu \neq 0)$. If $\nu=0$, the second term in (2.14), which arises from the double spectrum correction term in Eq. (2.7), goes like $t^{-1 / 2}$. This sudden change in the asymptotic behavior of the second term is presumably related to the phenomenon of an accumulation of an infinite number of Regge poles on the line $\operatorname{Re} l=-\frac{1}{2}$ at $\nu=0 .{ }^{4}$

In potential theory it is known that $\alpha(\infty) \leq-1$ for the class of potentials leading to a Mandelstam representation, hence the consistency requirement, Eq. (2.12) is satisfied. In the relativistic case, if $\alpha$ approaches a limit for large $\nu$, we require $\alpha(\infty)<-\frac{1}{2}+\epsilon$.

We conclude this section by remarking that the treatment of the two other spectral region contributions (in the relativistic problem) leads to the same results as obtained here, only with an appropriate permutation of the variables $s, t, u$. This is an obvious consequence of the equal-mass problem symmetry.

\section{UNEQUAL-MASS PROBLEM}

In this section we repeat the argument of Sec. II for an unequal-mass problem, such as pion-nucleon scattering (treated for simplicity without spin complications). We choose to look at an amplitude which has only an $s-u$ double spectral function. This is done because of the current interest in backward pion-nucleon scattering ${ }^{5}$ which one expects to be controlled by the $s-u$ spectral region. We shall speak briefly later about the other regions.

We continue to consider the contribution of Regge poles in the $s$ channel and will be ultimately interested in the behavior of the scattering amplitude for large $u$.

3a Note added in proof. We have assumed in this discussion that $\gamma(\infty)$ is a constant. If, in fact, $\gamma(s) \sim \nu^{-n}$ for large $\nu$, our condition on $\alpha(\infty)$ becomes relatively harmless, $\alpha(\infty)<n-\frac{1}{2}+\epsilon$.

${ }^{4}$ V. N. Gribov and I. Ya. Pomeranchak, Phys. Rev. Letters 9, 238 (1962)

${ }^{5}$ H. Brody et al., Phys. Rev. Letters 16, 828 (1966). 
The Regge-Sommerfeld-Watson representation which is defined originally in the physical $s$-channel region $s>s_{0}$ $=(M+\mu)^{2},-\infty<u<(M-\mu)^{2}$ is given by

$$
\begin{aligned}
A(s, u)=B(s, u)+\sum_{i} \gamma_{i}(s)(\nu)^{\alpha_{i}(s)} Q_{-1-\alpha_{i}(s)} & \\
& \times\left(-1+\frac{\left(r^{2} / s\right)-u}{2 \nu}\right),
\end{aligned}
$$

where $r=M^{2}-\mu^{2}$. As in the equal-mass case, $\gamma_{i}(s)(\nu)^{\alpha_{i}(s)}$ $=\left[2 \alpha_{i}(s)+1\right] \beta_{i}(s) / \cos \pi \alpha_{i}(s)$, where $\beta_{i}(s)$ is the residue of the Regge pole located at $l=\alpha_{i}(s)$; the variable $\nu$ is again the square of the center-of-mass momentum which is related to $s$ by

$$
\nu=\left[s-(M-\mu)^{2}\right]\left[s-(M+\mu)^{2}\right] / 4 s .
$$

Note that $\nu$ has zeros at $s=(M \pm \mu)^{2}$ and a pole at $s=0$; these properties play an important role in the derivation of a Regge formula with the correct Mandelstam analyticity. We shall assume in the subsequent discussion that $\gamma_{i}(s)$ and $\alpha_{i}(s)$ have only the physical threshold branch point at $s=s_{0 .}{ }^{6}$ We recall that the double spectral function for $A(s, u)$ has asymptotic boundaries at $s=(M+\mu)^{2}$ for $u \rightarrow \infty$ and $u=(M+\mu)^{2}$ for $s \rightarrow \infty$. As before, the summation in Eq. (3.1) runs over all Regge poles that appear in the region $\operatorname{Re} l>-\frac{1}{2}+\epsilon$ for any $s>s_{0}$. The background function $B(s, u)$ has the asymptotic property

$$
B(s, u)<\operatorname{const} \times u^{-\frac{1}{2}+\epsilon}, \quad u \rightarrow \infty
$$

for all positive $s>s_{0}$.

We begin our study by again using a one-dimensional dispersion relation (with fixed negative $u$ ) in order to obtain a representation of $A(s, u)$ valid for all $s$ :

$$
\begin{aligned}
A(s, u) & =\frac{1}{\pi} \int_{s_{0}}^{\infty} \frac{d s^{\prime}}{s^{\prime}-s} \operatorname{Im} B\left(s^{\prime}, u\right)+\frac{1}{\pi} \int_{s_{0}}^{\infty} \frac{d s^{\prime}}{s^{\prime}-s} \\
& \times \operatorname{Im}\left[\gamma\left(s^{\prime}\right)\left(\nu^{\prime}\right)^{\alpha} Q_{-1-\alpha}\left(-1+\frac{\left(r^{2} / s^{\prime}\right)-u}{2 \nu^{\prime}}\right)\right],
\end{aligned}
$$

where we have suppressed the summation over poles. The Regge-pole term in Eq. (3.4) can be written as a contour integral and evaluated:

$$
\begin{aligned}
A_{R}(s, u)= & \frac{1}{2 \pi i} \int_{c} \frac{d s^{\prime}}{s^{\prime}-s} \gamma\left(s^{\prime}\right)\left(\nu^{\prime}\right)^{\alpha} Q_{-1-\alpha}\left(-1+\frac{\left(r^{2} / s^{\prime}\right)-u}{2 \nu^{\prime}}\right) \\
= & \gamma(s)(\nu)^{\alpha} Q_{-1-\alpha}\left(-1+\frac{\left(r^{2} / s\right)-u}{2 \nu}\right) \\
& +\frac{1}{2} \int_{0}^{r^{2} / u} \gamma\left(s^{\prime}\right)\left(-\nu^{\prime}\right)^{\alpha} P_{\alpha}\left(1-\frac{\left(r^{2} / s^{\prime}\right)-u}{2 \nu^{\prime}}\right),
\end{aligned}
$$

where the contour $C$ encloses the threshold cut in a

\footnotetext{
${ }^{6}$ One arrives at this result by means of a generalization of the techniques employed by R. Oehme and G. Tiktopoulos [Phys. Letters 2, $86(1962)]$ for the equal-mass problem. Some care must be taken in studying the analytic properties at the point $s=0$.
}

clockwise manner. The second term in Eq. (3.5) comes from a left-hand cut (for negative $u$ ) in the integrand of the contour integral and is not present in the equalmass case [compare Eq. (2.5)]. It is perhaps worth noting that the derivation of Eq. (3.5), although straightforward in principle, is not entirely trivial.

By analogy with the equal-mass problem, we analytically continue $A_{R}(s, u)$ to the region $0<s<s_{0}$ and $u \gg 0$; we then compute the $u$ absorptive part:

$$
\begin{aligned}
D_{u}(u, s)= & \operatorname{Im} A_{R}(s, u)=-\gamma(s)(-v)^{\alpha}(\sin \pi \alpha) \\
& \times Q_{-1-\alpha}\left(-1+\frac{\left(r^{2} / s\right)-u}{2 \nu}\right)-\frac{1}{2} \int_{0}^{r 2 / u} \frac{d s^{\prime}}{s^{\prime}-s} \\
& \times \gamma\left(s^{\prime}\right)\left(v^{\prime}\right)^{\alpha} \sin \pi \alpha P_{\alpha}\left(1-\frac{\left(r^{2} / s^{\prime}\right)-u}{2 \nu^{\prime}}\right) .
\end{aligned}
$$

The function $D_{u}(u, s)$ for $u$ for $u \gg 0$ is a real analytic function $s$ with the threshold cut $s_{0}<s<\infty$ but with an unwanted cut $-\infty<s<\Sigma-u$, where $\Sigma=2\left(M^{2}+\mu^{2}\right)$. We remove the latter and also correct for the boundary of the double spectral region (since $D_{u}$ should be cut from $s_{0}$ to $\infty$ only as $\left.u \rightarrow \infty\right)$. This gives finally for the corrected absorptive part $\widetilde{D}_{u}$,

$$
\begin{aligned}
\widetilde{D}_{u}= & D_{u}-\frac{1}{2} \int_{-\infty}^{\Sigma-u} \frac{d s^{\prime}}{s^{\prime}-s} \gamma\left(s^{\prime}\right)\left(-\nu^{\prime}\right)^{\alpha} \\
& \times \sin \pi \alpha P_{\alpha}\left(-1+\frac{\left(r^{2} / s^{\prime}\right)-u}{2 \nu^{\prime}}\right)-\frac{1}{\pi} \int_{s_{0}}^{s_{0}+d / u} \frac{d s^{\prime}}{s^{\prime}-s} \\
& \times \operatorname{Im}\left[-\gamma\left(s^{\prime}\right) \nu^{\prime \alpha} \sin \pi \alpha Q_{-1-\alpha}\left(1-\frac{\left(r^{2} / s^{\prime}\right)-u}{2 \nu^{\prime}}\right)\right] .
\end{aligned}
$$

The upper limit on the last term in Eq. (3.7), which is the one that corrects the double spectral function boundary, is correct only for $u \rightarrow \infty$; one can, of course, use the exact curve in any given situation.

The Regge-pole term $R(s, u)$ which has the correct analyticity is defined as

$$
R(s, u)=\frac{1}{\pi} \int_{u_{1}}^{\infty} \frac{d u^{\prime}}{u^{\prime}-u} \widetilde{D}_{u}\left(u^{\prime}, s\right) .
$$

The lower limit $u_{1}$ may have any value greater than the $u$ threshold of $(M+\mu)^{2}$. In writing Eq. (3.7) we have assumed that $u>\Sigma$ so that we must have $u_{1}>\Sigma$. Smaller values of $u_{1}$ may be used but one must then analytically continue Eq. (3.7). In practice we are interested in $R(s, u)$ for large $u$ so that $u_{1} / u \ll 1$ and the precise value is irrelevant.

The discussion of asymptotic behavior and consistency will be taken up in the next section.

\section{ASYMPTOTIC BEHAVIOR AND CONSISTENCY IN UNEQUAL-MASS PROBLEM}

In this section we emphasize the important differences between the equal- and unequal-mass Regge formulas 
[we refer here to our modifications which incorporate the analyticity requirements, Eqs. (2.8) and (3.8)] with regard to asymptotic behavior $(u \rightarrow \infty$ in the present case) and the consistency of Mandelstam and $l$-plane analyticity. The issue of asymptotic behavior is dramatically different in unequal-mass scattering for purely kinematical reasons: The cosine of the scattering angle for the $s$ reaction $z_{s}$ does not become large for all fixed $s$ as $u \rightarrow \infty$ in the physical region of the $u$ reaction. To see this we recall that

$$
z_{s}=1+\frac{t}{2 \nu}=1+\frac{\Sigma-s-u}{2 \nu}=-1+\frac{\left(r^{2} / s\right)-u}{2 \nu} .
$$

The physical region for the $u$ channel includes the positive $s$ domain $0 \leq s<r^{2} / u, u \geq(m+\mu)^{2}$. Evidently, no matter how large $u$ becomes in the $u$-channel physical region (it is of course limited by $u \leq r^{2} / s$ ), $\left|z_{s}\right| \leq 1$ in this region. At the value $s=0, u$ can become indefinitely large, but $z_{s}=+1$ and the question of interest is whether the familiar Regge asymptotic form which holds for $s<0, u \rightarrow \infty$, namely, $u^{\alpha(s)}$, persists at $s=0$.

It is perhaps worth noting that from the simpleminded Regge point of view we require $\left|z_{s}\right| \gg 1$, which in the neighborhood $s=0$ means $u \gg r^{2} /|s|$. This peculiar nonuniform approach to the asymptotic regime is, as we shall show, a fault of the Regge representation which is corrected by incorporating the correct analyticity. The fact that there is no singularity in the whole amplitude at $s=0$ suggests, but of course does not prove, that an asymptotic form valid for $s<0$ persists at $s=0$; an asymptotic expansion of an analytic function does not necessarily share the analyticity of the original function.

The question of consistency of Mandelstam and $l$ plane analyticity is also changed in the unequal-mass problem because of the presence of the integral term over the region $0<s<r^{2} / u$ in the absorptive part $D_{u}(u, s)$ given by Eq. (3.6). This term has no counterpart in the equal-mass case where $D_{t}(t, \nu), \mathrm{Eq} .(2.6)$, is entirely given by the $Q$ function.

The consistency issue and asymptotic behavior are so closely linked that we discuss them more or less together. To begin, we define a correction function $C(s, u)$ by analogy with Eq. (2.10) as follows:

$$
\begin{aligned}
C(s, u)=R(s, u)-\gamma(s)(\nu)^{\alpha(s)} & Q_{-1-\alpha(s)} \\
& \times\left(-1+\frac{\left(r^{2} / s\right)-u}{2 \nu}\right) .
\end{aligned}
$$

We then require for consistency [at least for $s>s_{0}$ where
Eq. (3.1) is originally defined] that

$$
C(s, u) \leq \text { const } u^{-\frac{1}{2}+\epsilon}, \quad u \rightarrow \infty .
$$

Again, to within terms of order $u^{-1}$ this consistency check can be made by simply using $\widetilde{D}_{u}(u, s)$. The terms in $\widetilde{D}_{u}$ that must be examined are all those apart from

$$
-\gamma(s)(-\nu)^{\alpha} \sin \pi \alpha Q_{-1-\alpha}\left(-1+\frac{\left(r^{2} / s\right)-u}{2 \nu}\right) .
$$

From Eqs. (3.6) and (3.7) we see that there are three such terms. We first inspect the difference $\widetilde{D}_{u}-D_{u}$ which consists of the two integral terms in Eq. (3.7). The evaluation of $\widetilde{D}_{u}-D_{u}$ is very similar to that discussed in Sec. II for $\widetilde{D}_{t}-D_{t}$, Eq. (2.14). We find

$$
\tilde{D}_{u}-D_{u} \sim D_{1} u^{\alpha(\infty)}+D_{2} u^{-3 / 2}, \quad\left(u \rightarrow \infty, s \neq s_{0}\right),
$$

where $D_{1}$ and $D_{2}$ are functions of $s$. The second term changes character abruptly at $s=s_{0}$ and becomes $\sim u^{-1 / 2}$. Provided that it is reasonable to discuss the consistency question for each double spectral region separately, we would again require $\alpha(\infty)<-\frac{1}{2}+\epsilon$ as in the equal-mass case.

Finally, we discuss the asymptotic behavior of $D_{u}(u, s)$, Eq. (3.6). The integral term in $D_{u}$ should also be of background size for consistency. It is useful to rewrite $D_{u}(u, s)$ in a form which will also be convenient for examining the asymptotic behavior in $u$ for $s=0$. The point is that although both terms in Eq. (3.6) diverge like $s^{-\alpha(0)}(\ln s)$ near $s=0$, the sum is, in fact, analytic at this point by construction. We wish to make the cancellation of singularities manifest.

To proceed, we use the following dispersion relation:

$$
\begin{gathered}
\gamma(0)\left(\frac{-r^{2}}{4 s}\right)^{\alpha(0)}[\sin \pi \alpha(0)] Q_{-1-\alpha(0)}\left(1-\frac{2 s u}{r^{2}}\right) \\
=\gamma(0)[\sin \pi \alpha(0)]\left[-\frac{1}{2} \int_{0}^{r^{2} / u} \frac{d s^{\prime}}{s^{\prime}-s}\left(\frac{r^{2}}{4 s^{\prime}}\right)^{\alpha(0)} P_{\alpha(0)}\right. \\
\left.\times\left(-1+\frac{2 s^{\prime} u}{r^{2}}\right)+\frac{\pi^{1 / 2} \Gamma(-\alpha(0))}{\Gamma\left(-\alpha(0)-\frac{1}{2}\right)} u^{\alpha(0)}\right]
\end{gathered}
$$

where the term proportional to $u^{\alpha(0)}$ is a subtraction constant. The left-hand side of $\mathrm{Eq}$. (4.5) gives the leading singular behavior near $s=0$ of the first term in $D_{u}$, Eq. (3.6). The utility of this form is that an explicit cancellation of the singularity in the second term of (3.6) can be made. We find then for $D_{u}(u, s)$ the following expression :

$$
\begin{aligned}
& D_{u}(u, s)=-\gamma(0)[\sin \pi \alpha(0)] \frac{\pi \Gamma(-\alpha(0))}{\Gamma\left(-\alpha(0)+\frac{1}{2}\right)} u^{\alpha(0)}- {\left[\gamma(s)(-\nu)^{\alpha(s)}[\sin \pi \alpha(s)] Q_{-1-\alpha(s)}\left(-1+\frac{\left(r^{2} / s\right)-u}{2 \nu}\right)\right.} \\
&\left.-\gamma(0)\left(-\frac{r^{2}}{4 s}\right)^{\alpha(0)}[\sin \pi \alpha(0)] Q_{-1-\alpha(0)}\left(1-\frac{2 s u}{r^{2}}\right)\right]-\frac{1}{2}\left\{\int _ { 0 } ^ { r ^ { 2 } / u } \frac { d s ^ { \prime } } { s ^ { \prime } - s } \left[\gamma\left(s^{\prime}\right) \nu^{\prime \alpha\left(s^{\prime}\right)}\left[\sin \pi \alpha\left(s^{\prime}\right)\right] P_{\alpha\left(s^{\prime}\right)}\right.\right. \\
&\left.\left.\quad \times\left(1-\frac{\left(r^{2} / s^{\prime}\right)-u}{2 \nu^{\prime}}\right)-\gamma(0)\left(\frac{r^{2}}{4 s^{\prime}}\right)^{\alpha(0)}[\sin \pi \alpha(0)] P_{\alpha(0)}\left(-1+\frac{2 s^{\prime} u}{r^{2}}\right)\right]\right\} .
\end{aligned}
$$


Let us now examine $D_{u}(u, s)$ for large $u$. If $s \neq 0$, the $u^{\alpha(0)}$ term is cancelled by the $Q_{-1-\alpha(0)}$ term and we find

$$
\begin{aligned}
D_{u}(u, s) \sim-\gamma(s)(-\nu)^{\alpha(s)} Q_{-1-\alpha(s)} & \left(-1+\frac{\left(r^{2} / s\right)-u}{2 \nu}\right) \\
& +\operatorname{const} \times u^{\alpha(0-1},
\end{aligned}
$$

as $u \rightarrow \infty$. The term in curly brackets in (4.6) goes like $u^{\alpha(0)-2}$. In order to have consistency, the second term on the right of Eq. (4.7) should be of background size, i.e.,

$$
\begin{aligned}
\alpha(0)-1 & \leq-\frac{1}{2}+\epsilon, \\
\alpha(0) & \leq \frac{1}{2}+\epsilon, \quad 0<\epsilon<\frac{1}{2} .
\end{aligned}
$$

This condition is undoubtedly fulfilled in the interesting case of pion-nucleon scattering. We defer further discussion of the general situation to the next section.

Now we come to the question of the asymptotic behavior at $s=0$. The two terms in the first square bracket of Eq. (4.6) cancel in this limit whereas the terms in curly brackets yield a term proportional to $u^{\alpha(0)-1}$. We are left then with the first term $u^{\alpha(0)}$, which is precisely what would have been obtained from a blind extrapolation of the Regge behavior valid for large $u$ and $s<0$. The way this has come about from our enforced analyticity is remarkable.

There remains finally the question of the behavior of the amplitude for fixed positive $s<(M-\mu)^{2}$. There is in this region no possibility of $u$ becoming indefinitely large and thus one cannot talk about an asymptotic form. It is a quantitative question whether the Regge term we have isolated is large compared to the background. It is clear, however, that if we sit on the curve $s=r^{2} / u$, as $u$ increases, we will recover our behavior $u^{\alpha(0)}$.

Before closing this section we remark that an entirely analogous treatment of the $s-t$ spectral function can be readily given in the unequal-mass problem and one finds an asymptotic behavior of $(-u)^{\alpha(0)}$ at $s=0$ as $u \rightarrow+\infty$. Because of the different analyticity structure, there is no tendency for cancellation between the $s-u$ and $s-t$ spectral region contributions. The term $(-u)^{\alpha(0)}$ would be automatically included if we introduced angularmomentum amplitudes of definite signature into the problem.

\section{CONCLUSIONS AND DISCUSSION}

We have studied the consequences of requiring simultaneously $l$-plane and Mandelstam analyticity for twoparticle elastic-scattering amplitudes. We assume that the partial-wave amplitude is meromorphic in the $l$ plane to the right of Rel $=-\frac{1}{2}+\epsilon$, where $0<\epsilon<\frac{1}{2}$. Then we insist that the Mandelstam-Regge-SommerfeldWatson representation implied by the $l$-plane analyticity should be consistent with the Mandelstam double dispersion representation. We perform surgery on the isolated Regge-pole contributions to make them conform with Mandelstam analyticity and investigate whether the required correction terms are of a magnitude that can be cancelled by the background integral. We furthermore assume that each spectral region may be handled separately.

When this procedure is applied to the scattering of equal-mass particles (or in potential theory), we find that a necessary constraint of the Regge trajectory $\alpha(s)$ is that $\alpha(\infty)$ be negative. We obtain a modified Regge representation in which the pole terms have the proper spectral support and which, when all three spectral regions are included, provides a suitable form for either phenomenological analysis or as an ansatz for dynamical calculations of the variety discussed by Chew and Jones. ${ }^{1}$

The corresponding treatment of unequal-mass scattering amplitudes was considerably more complicated and has some implications which may be of a rather fundamental nature. One of the features of unequalmass problems is that the cosine of the scattering angle in the crossed channel does not become large at high energies (and in fact for part of the physical region lies between \pm 1 no matter how large the energy). This has led to considerable uneasiness in application of Reggepole ideas to such processes. We have found from our representation that the Regge asymptotic form $u^{\alpha(s)}$ valid for $s<0, u \rightarrow \infty$ persists down to $s=0$ even though $\cos \theta_{s} \rightarrow+1$. This comes about by virtue of the fact that one of the correction terms forced by analyticity takes over the asymptotic behavior previously given for $s<0$ by the usual Regge term $Q_{-1-\alpha}\left(\cos \theta_{s}\right)$.

The persistence of Regge asymptotic behavior near $s=0$ was conjectured by Chew and $\mathrm{Stack}^{7}$ and the question has recently been studied by Freedman and Wang, ${ }^{8}$ who use techniques quite different from ours. These authors urge the use of a representation of scattering amplitudes directly in terms of powers, in a manner first suggested by Khuri, ${ }^{9}$ so that the cosine of the scattering angle in the crossed channel never appears. We feel that this approach, while leading rather easily to results about asymptotic behavior, is less satisfactory than the one we have presented here. If one attempts to describe the power-law behavior in these terms, he finds that $u^{\alpha(s)}$ in the $l$ plane has poles at $l=\alpha(s), \alpha(s)-1, \alpha(s)-2, \cdots$, which can produce difficulties. ${ }^{10}$ Our representation, incorporating as it does all the correct analyticity and threshold properties as well as involving only individual Regge poles (and no satellites at $\alpha-1$, etc.), is hopefully suitable for discussion of the direct channel amplitude at low energies. This means that a representation (generalized to include

${ }^{7}$ J. D. Stack, Phys. Rev. Letters 16, 286 (1966); see also G. F. Chew and J. D. Stack, University of California Laboratory Report No. UCRL-16293 (unpublished).

${ }^{8}$ D. Freedman and J. M. Wang, Phys. Rev. Letters 17, 569 (1966).

${ }_{9}$ N. Khuri, Phys. Rev. Letters 10, 420 (1963); Phys. Rev. 132, 914 (1963).

${ }^{10}$ For a discussion of some drawbacks of the Khuri representation see C. E. Jones, Phys. Rev. 135, B214 (1964). 
all spectral regions) based on our results can be expected to give a reasonable description of the scattering amplitude at both high and low energies. The recent analysis of backward pion-nucleon scattering by Barger and Cline ${ }^{11}$ lends support to this idea. We feel, however, that the form of their Regge-pole term is of a somewhat ad hoc character. It would be interesting to compare the energy dependence of their Regge-pole term with that predicted by our Eq. (3.8), which should be more precise.

We turn finally to the question of the consistency of $l$-plane and Mandelstam analyticity in the unequal-mass problem. As in the equal-mass case we must require that $\alpha(\infty)$ be negative. The more striking requirement is that $\alpha(0)$ be less than $\frac{1}{2}+\epsilon, 0<\epsilon<\frac{1}{2}$, which arises from the presence of the asymptotic term $u^{\alpha(0)-1}$. If $\alpha(0)<\frac{1}{2}$, there is no problem. If, however, $\alpha(0)>\frac{1}{2}$ and if $\epsilon$ can take on any value greater than zero but less than onehalf, one has a contradiction, for there would be nothing to cancel the fixed singularity in the angular-momentum plane at $l=\alpha(0)-1$. It is conceivable that in this case such a singularity would be covered by cuts in the angular-momentum plane, the possible existence of which has been ignored. A very subtle failure of the Mandelstam representation for unequal masses is not

${ }^{11}$ V. Barger and D. Cline, Phys. Rev. Letters 16, 913 (1966). out of the question. If there were an infinite number of Regge poles to the right of $\operatorname{Re} l=-\frac{1}{2}$ in the relativistic problem, the term $u^{\alpha(0)-1}$ might be cancelled. ${ }^{11 a}$

Finally, we note that the requirement that $\alpha(\infty)$ be negative, which holds in both the equal- and unequalmass problems, implies in particular for the Pomeranchuk trajectory the existence of ghost states.

We have no further light to shed on these points but wish to draw attention to the fact that the constraints on the asymptotic behavior of scattering amplitudes implied by combined $l$-plane and Mandelstam analyticity may be of fundamental importance.

\section{ACKNOWLEDGMENT}

It is a pleasure to thank Dr. M. Froissart for several stimulating conversations.

11a Note added in proof. An alternate explanation based on the existence of a family of trajectories $\alpha_{k}(s)$ [with residue functions $\left.\gamma_{k}(s)\right]$ arranged so that $\alpha_{k}(0)=\alpha(0)-k, k=1,2, \cdots$, and $\gamma_{k} \sim s^{-k}$ near $s=0$ has been proposed by Freedman and Wang (Ref. 8). An improved version of their argument based on the formalism of the present paper has been given by one of us (C.E.J.) in collaboration with Freedman and Wang (to be published). It should be emphasized that the existence of this family of trajectories has been demonstrated only in a simple model; we have no real assurance that this way of avoiding the conflict between Mandelstam and angular momentum analyticity has been chosen by nature. 